\title{
Efficient Light Emitters in the Solid State: Synthesis, Aggregation-Induced Emission, Electroluminescence, and Sensory Properties of Luminogens with Benzene Cores and Multiple Triarylvinyl Peripherals
}

\author{
Carrie Y. K. Chan, Zujin Zhao, Jacky W. Y. Lam, Jianzhao Liu, Shuming Chen, Ping Lu, \\ Faisal Mahtab, Xiaojun Chen, Herman H. Y. Sung, Hoi Sing Kwok, Yuguang Ma, \\ Ian D. Williams, Kam Sing Wong, and Ben Zhong Tang**
}

Benzene-cored luminogens with multiple triarylvinyl units are designed and synthesized. These propeller-shaped molecules are nonemissive when dissolved in good solvents, but become highly emissive when aggregated in poor solvents or in the solid state, showing the novel phenomenon of aggregation-induced emission. Restriction of intramolecular motion is identified as the main cause for this effect. Thanks to their high solid-state fluorescence quantum yields (up to unity) and high thermal and morphological stabilities, light-emitting diodes with the luminogens as emitters give sky-blue to greenishblue light in high luminance and efficiencies of $10800 \mathrm{~cd} \mathrm{~m}^{-2}, 5.8 \mathrm{~cd} \mathrm{~A}^{-1}$, and $2.7 \%$, respectively. The emissions of the nanoaggregates of the luminogens can be quenched exponentially by picric acid, or selectively by $\mathrm{Ru}^{3+}$, with quenching constants up to $10^{5}$ and $\sim 2.0 \times 10^{5} \mathrm{~L} \mathrm{~mol}^{-1}$, respectively, making them highly sensitive (and selective) chemosensors for explosives and metal ions.

\section{Introduction}

Scientists and technologists have devoted much effort to the development of sensitive and selective sensory systems for the detection of chemicals, metals, and biopolymers, because they play important roles in industrial, environmental, and biological processes and systems. Sensors based on fluorescent materials have attracted special attention, as they offer ultrahigh sensitivity, ultralow detection limits, great interference tolerance, and specific-analyte targeting, in addition to their ultrafast response, simple operation, field portability, and in-situ workability. ${ }^{[1]}$ In response to the demand, a large number of luminescent molecules have been synthesized. Many are highly emissive in dilute solutions, with fluorescence quantum yields approaching unity. On the other hand, many chemical and biological analytes are ionic species existing or working in aqueous media. Incongruously, however, most fluorophores are hydrophobic aromatics that are barely soluble in water. Although their water miscibility can be improved by incorporating hydrophilic groups, the resultant amphiphilic molecules tend to aggregate when dispersed in aqueous media. Formation of aggregates often partially-and sometimes even totally-quenches the light emission. This notorious effect of aggregation-caused quenching (ACQ) has led fluorophores to be studied as isolated molecules in very dilute solutions. ${ }^{[2]}$ The use of dilute solutions, however, leads to poor sensitivity in fluorescence sensory systems. Inorganic quantum dots can surmount some of the disadvantages of the organic fluorophores, but they also pose new problems, such as the difficulty of their syntheses, the limited varieties, and their inherently high toxicities. ${ }^{[3]}$

In our research for efficient light-emitting materials, we were attracted by a group of novel molecules called siloles. These molecules are practically nonfluorescent when dissolved in good solvents as isolated species, but become highly emissive when aggregated into nanoparticles in poor solvents (e.g., water) or fabricated into thin films in the solid state. ${ }^{[4]}$ We coined the 
term "aggregation-induced emission (AIE)" for this phenomenon because the nonemissive silole molecules do emit on formation of aggregates. Recently, we and other groups found that other conjugated compounds also exhibit AIE activity. ${ }^{[5]}$ For example, while trans-1,4-distyrylbenzene is a traditional ACQ fluorophore, Diau, Hsu, and co-workers found that attaching two methyl groups to the double bonds of 1,4-distyrylbenzene can transform it into an AIE fluorogen. ${ }^{[6]} \mathrm{Wu}$ et al. observed marked AIE effects for a series of $8,8 \mathrm{a}$-dihydrocyclopenta[a] indene derivatives. ${ }^{[7]}$ Tao et al. reported the AIE feature of a $\Lambda$-shaped pyridinium salt. ${ }^{[8]}$ Wang and co-workers integrated benzobis(thiadiazole) with triphenylamine and generated an AIE-active molecule with an emission maximum in the nearIR region. ${ }^{[9]}$ Chujo and co-workers observed a similar AIE effect in polymers with $o$-carborane and $p$-phenyleneethynylene sequences. ${ }^{[10]} \mathrm{Li}$ and Park reported a series of $\operatorname{Ir}(\mathrm{III})$ complexes with aggregation-induced phosphorescence characteristics. ${ }^{[1]}$ The AIE systems developed so far range from hydrocarbons to organometallics, from small molecules to big polymers, and from extensively conjugated systems to those without typical chromophores, which is demonstrative of the ubiquity of the AIE effect.

AIE fluorogens have been used for the fabrication of efficient optoelectronic devices. ${ }^{[12]}$ In contrast, much less work has been carried out on their applications in environmental and biological sciences. ${ }^{[13]}$ In this paper, we aim to synthesize new AIE luminogens with potential applications in these fields. We prepared benzene-cored luminogens carrying multiple triarylvinyl peripherals and found that they were all AIE-active with high solid-state-fluorescence quantum yields. Organic light-emitting diodes (OLEDs) based on the luminogens were constructed, which showed sky-blue and greenish-blue light in high luminance and efficiency. These emissions could be faded by picric acid, and selectively by $\mathrm{Ru}^{3+}$, with large quenching constants, which renders them promising chemosensors for explosives and metal ions.

\section{Results and Discussion}

\subsection{Synthesis}

To enrich the AIE research and broaden its practical applications, we designed three new AIE luminogens and devised a multistep reaction route for their syntheses (Scheme 1). The Friedel-Crafts acylation of terephthaloyl chloride (1) with benzene furnished 2, which was converted into TPTPE by reaction with diphenylmethyl lithium (4) followed by acid-catalyzed dehydration. Using similar synthetic procedures, we also prepared TPTDPE and BTPTPE with thiophene and additional 1,2,2-triphenylvinyl substituents, respectively.

All intermediates and final products were carefully purified and fully characterized by NMR and mass spectroscopies, which confirmed their expected molecular structures. Single crystals of TPTPE, TPTDPE, and BTPTPE were isolated from their dichloromethane (DCM)/methanol solutions, and were characterized crystallographically. Their ORTEP drawings are shown in Figure 1, while Table 1 and Table S1-S3 in the Supporting Information summarize the crystal data. Note that Wang had prepared TPTPE by a three-step synthetic route but failed to obtain its crystal structure. ${ }^{[14]}$ Instead of investigating its optical and electrochemical properties, however, no effort was made on the exploration of its practical applications. All the luminogens were soluble in common organic solvents, such as tetrahydrofuran (THF), toluene, DCM, and chloroform, but were insoluble in water.
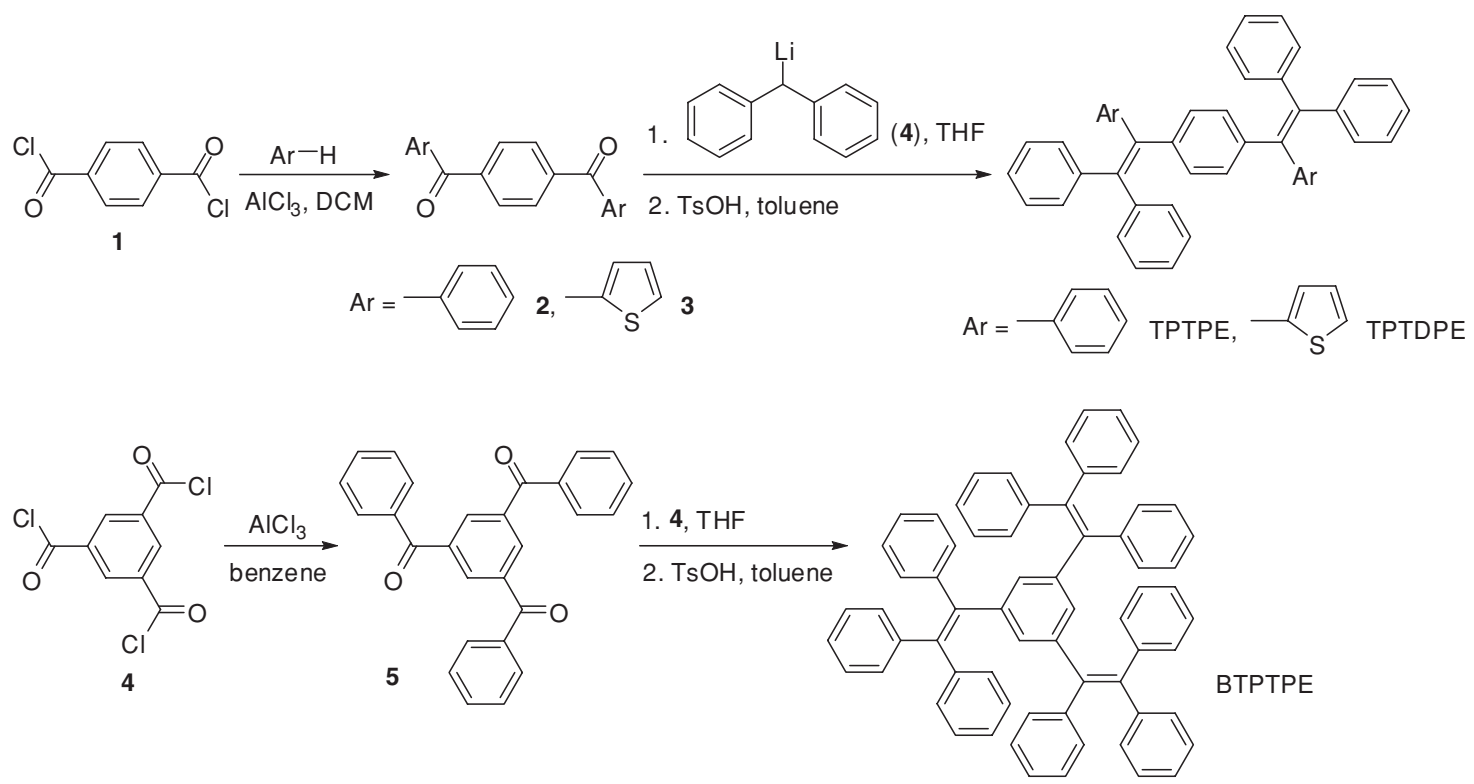

Scheme 1. Synthetic routes to TPTPE, TPTDPE, and BTPTPE. 

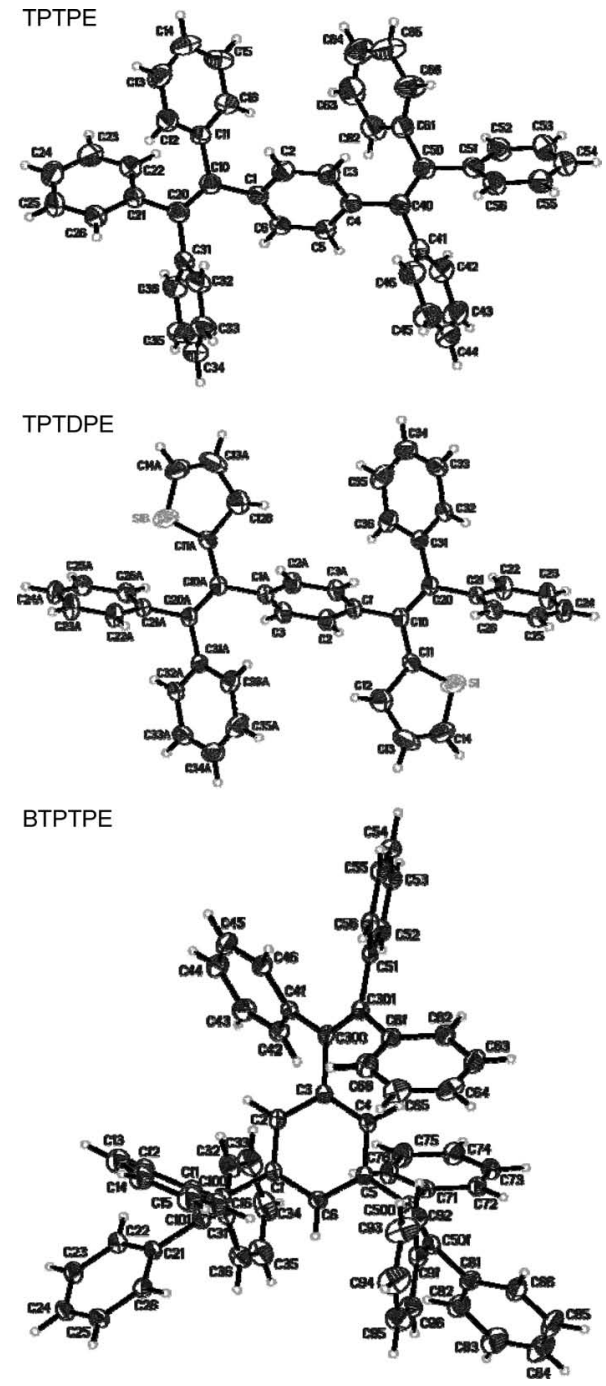

Figure 1. ORTEP drawings of TPTPE, TPTDPE, and BTPTPE.

\subsection{Optical Properties}

Figure 2 shows the absorption spectra of the benzene-cored luminogens in dilute THF $(10 \mu \mathrm{M})$. The ultraviolet (UV) spectra of TPTPE and TPTDPE are similar, with peak maxima at 326 and $332 \mathrm{~nm}$, respectively. The spectrum of BTPTPE, however, is blueshifted relative to those of TPTPE and TPTDPE, albeit at a higher absorptivity, which suggests that it possesses lower conjugation. This can be understood because the triarylvinyl units of TPTPE and TPTDPE interact electronically on the same plane via the benzene core, whereas those of BTPTPE are metaconjugated, which leads to a shorter effective conjugation length in BTPTPE and hence absorption at the shorter wavelengths.

The photoluminescence (PL) spectra of TPTPE, TPTDPE, and BTPTPE in dilute THF $(10 \mu \mathrm{M})$ exhibit only noisy PL signals with no discernible peak maxima (Figure 3 and Figure S1 in the Supporting Information). The fluorescence quantum yields $\left(\Phi_{\mathrm{FS}}\right)$ of TPTPE, TPTDPE, and BTPTPE, estimated using 9,10-diphenylanthracene as standard, are merely $0.22,0.11$, and
$0.09 \%$, respectively, which indicates that they are practically nonluminescent when dissolved in good solvents.

To investigate whether the new luminogens are AIE-active, we added water, a nonsolvent for the luminogens, to the THF solutions and monitored the PL change. The PL intensity of TPTPE remains low in aqueous mixtures with water content less than $70 \%$, above which it increases swiftly. From the pure THF solution to $\mathrm{THF} / \mathrm{H}_{2} \mathrm{O}$ mixture with $90 \%$ water content, the PL intensity rises 1000 fold. Particle-size analysis of the aqueous mixtures with high water content confirms the formation of nanoparticles with average sizes of 120-200 nm. Clearly, the PL of TPTPE is enhanced by aggregate formation; in other words, it is AIE-active. The AIE phenomenon is not an isolated case observed only in TPTPE but is also found in TPTDPE and BTPTPE.

Closer inspection of the PL spectra of BTPTPE in aqueous mixtures reveals that the emission maximum is bathochromically shifted from 450 to $460 \mathrm{~nm}$, and that this shift is accompanied by a reduction in intensity when the water content is increased from 85 to 90 and then to $95 \%$.This is due to the morphological change in the aggregates from crystalline to amorphous: In solvent mixtures with low water content $(\leq 50 \%)$, the molecules steadily assemble into crystalline clusters, whereas in mixtures with high water fractions $(>85 \%)$, the molecules abruptly agglomerate into amorphous aggregates, as confirmed by transmission electron microscope (TEM) and electron diffraction (ED) measurements. Whilst clear diffraction spots are seen in the ED pattern of the aggregates of BTPTPE formed in $50 \%$ aqueous mixture, the aggregates formed in $95 \%$ aqueous mixture give only a diffuse halo (Figure 4).

To better understand the photophysical properties of the luminogens, we performed theoretical calculations on their energy levels. Their highest-occupied molecular orbital (HOMO) and lowest occupied molecular orbital (LUMO) plots are shown in Figure 5. The HOMO and LUMO of TPTPE and TPTDPE are dominated by the orbitals from the benzene core and the triarylvinyl units, which reveals that their absorption and emission stem from the $\pi-\pi *$ transitions and exciton decays of the whole molecules. However, the severe steric hindrance between the peripherals in BTPTPE leads to poorer electronic communication. As a result, the orbitals are mainly localized on the benzene core conjugated with one or two triphenylvinyl units. Such orbital distribution has somewhat shortened the effective conjugation length. The calculated bandgap for BTPTPE is $4.226 \mathrm{eV}$, which is wider than those of TPTPE and TPTDPE (4.018 and $3.695 \mathrm{eV}$, respectively). The theoretical study thus nicely explains the hypsochromic shifts in the absorption and emission of BTPTPE relative to those of TPTPE and TPTDPE.

We further investigated the PL behaviors of the luminogens in the solid state. The emissions of the TPTPE, TPTDPE, and BTPTPE films show a peak at $480-450 \mathrm{~nm}$, which is similar to the PLs of their aggregates in $\mathrm{THF} / \mathrm{H}_{2} \mathrm{O}$ mixtures (Table 2). In sharp contrast to their weak fluorescence in solution, all the luminogens emit strongly in the condensed phase, with $\Phi_{\mathrm{F}}$ values (measured by an integrating sphere) of 100, 25.5, and $100 \%$, respectively.

We proposed that the restriction of intramolecular rotation (IMR) is the main cause for the AIE. ${ }^{[15]}$ In dilute solution, the multiple aromatic rings in TPTPE, TPTDPE, and BTPTPE can 
Table 1. Summary of crystal data and reflection collection parameters for TPTPE, TPTDPE, and BTPTPE.

\begin{tabular}{|c|c|c|c|}
\hline & TPTPE & TPTDPE & BTPTPE \\
\hline empirical formula & $\mathrm{C}_{46} \mathrm{H}_{34}$ & $\mathrm{C}_{42} \mathrm{H}_{30} \mathrm{~S}_{2}$ & $\mathrm{C}_{67.75} \mathrm{H}_{52.50} \mathrm{Cl}_{2.50} \mathrm{O}_{0.50}$ \\
\hline formula weight & 586.73 & 598.78 & 963.22 \\
\hline crystal dimensions [mm] & $0.40 \times 0.15 \times 0.12$ & $0.30 \times 0.28 \times 0.15$ & $0.32 \times 0.25 \times 0.03$ \\
\hline crystal system & triclinic & monoclinic & monoclinic \\
\hline space group & $P-1$ & $P 2(1) / c$ & $P 2(1) / c$ \\
\hline$a[\AA ̊]$ & $10.129(3)$ & $12.1008(4)$ & $12.7233(3)$ \\
\hline$b[\AA]]$ & $12.382(4)$ & $9.3658(3)$ & $17.5697(3)$ \\
\hline$c[\AA]]$ & $14.475(5)$ & $14.6950(5)$ & $24.5934(5)$ \\
\hline$\alpha[\operatorname{deg}]$ & $99.928(5)$ & 90 & 90 \\
\hline$\beta[\operatorname{deg}]$ & $100.985(5)$ & $108.772(4)$ & $92.341(2)$ \\
\hline$\gamma[\mathrm{deg}]$ & $106.356(4)$ & 90 & 90 \\
\hline$V\left[\AA^{3}\right]$ & $1660.0(10)$ & $1576.85(9)$ & $5493.1(2)$ \\
\hline$Z$ & 2 & 2 & 4 \\
\hline$D_{\text {calcd. }}\left[\mathrm{g} \mathrm{cm}^{-3}\right]$ & 1.174 & 1.261 & 1.165 \\
\hline$F_{000}$ & 620 & 628 & 2022 \\
\hline Temp [K] & $298(2)$ & $173(2)$ & $133(2)$ \\
\hline Radiation $(\lambda),[\AA]$ & 0.71073 & 1.54178 & 1.54178 \\
\hline$\mu(\mathrm{Mo} \mathrm{K} \alpha)\left[\mathrm{mm}^{-1}\right]$ & 0.066 & 1.742 & 1.593 \\
\hline $2 \theta_{\max }[\mathrm{deg}]$ (completeness) & $25(98.7 \%)$ & $66.5(98.2 \%)$ & $66.5(98.2 \%)$ \\
\hline no. of collected reflns. & 15329 & 8133 & 30269 \\
\hline no. of unique reflns. $\left(R_{\text {int }}\right)$ & $6402(0.0396)$ & $2792(0.0331)$ & $9720(0.0724)$ \\
\hline data/restraints/parameters & $6402 / 0 / 415$ & $2792 / 0 / 199$ & $9720 / 4 / 649$ \\
\hline$R_{1}, \mathrm{w} R_{2}[\mathrm{obs} I>2 \sigma(I)]$ & $0.0547,0.0893$ & $0.0441,0.1225$ & $0.0641,0.1494$ \\
\hline$R_{1}, w R_{2}$ (all data) & $0.1130,0.0992$ & $0.0526,0.1274$ & $0.1033,0.1603$ \\
\hline residual peak/hole e. $\AA^{-3}$ & $0.161 /-0.121$ & $0.428 /-0.296$ & $0.647 /-0.672$ \\
\hline transmission ratio & $1.00 / 0.88$ & $1.00 / 0.76$ & $1.00 / 0.60$ \\
\hline goodness-of-fit on $F^{2}$ & 1.005 & 1.012 & 1.008 \\
\hline
\end{tabular}

undergo active IMR. Collectively, these multiple molecular motions effectively consume the photonic energy of the excited state as thermal energy, which consequently quenches the light emission of the luminogens in pure THF. In the aggregated or film state, the IMR process is restricted, which blocks the nonradiative relaxation pathway and populates radiative decay, thus turning the dye molecules into strong emitters.

Interestingly, the crystals of TPTPE, TPTDPE, and BTPTPE emit at 435-455 nm, which is $12-57 \mathrm{~nm}$ blueshifted from those of the amorphous films. Similar phenomena were also observed in other TPE derivatives. ${ }^{[16]}$ The unusual blueshift observed in the crystalline phase may be attributable to the conformation twisting of the aromatic rings of the luminogens to fit into the crystalline lattices. Without such restraint, the molecules in the amorphous state may assume a more planar conformation and thus show a redder luminescence.

To gain further insight into the AIE mechanism, we examined the geometric structures and packing arrangements of TPTPE, TPTDPE, and BTPTPE in the crystalline state. As shown in Figure 6, all the luminogens take a twisted conformation, which prevents the formation of species that are detrimental to light emission by intermolecular interactions. Multiple $\mathrm{C}-\mathrm{H} \cdots \pi$ hydrogen bonds with distances of $2.745-2.888 \AA$ are formed between the hydrogen atoms of the phenyl rings in one molecule and the $\pi$ clouds of the phenyl rings in another molecule. These multiple $\mathrm{C}-\mathrm{H} \cdots \pi$ hydrogen bonds help rigidify the molecular conformation and lock the molecular rotation. As a result, the excited-state energy consumed by IMR is greatly reduced, thus enabling the molecules to emit intensely in the solid state.

\subsection{Thermal Properties}

The thermal stability of TPTPE, TPTDPE, and BTPTPE was evaluated by thermogravimetric analysis (TGA) under nitrogen at a heating rate of $10{ }^{\circ} \mathrm{C} \mathrm{min}-1$. As shown in Figure 7, all the luminogens are thermally stable, losing $5 \%$ of their weights at temperatures $\left(T_{d}\right)$ from 293 to $338{ }^{\circ} \mathrm{C}$. Their phase transitions were investigated by differential scanning calorimetry (DSC) 


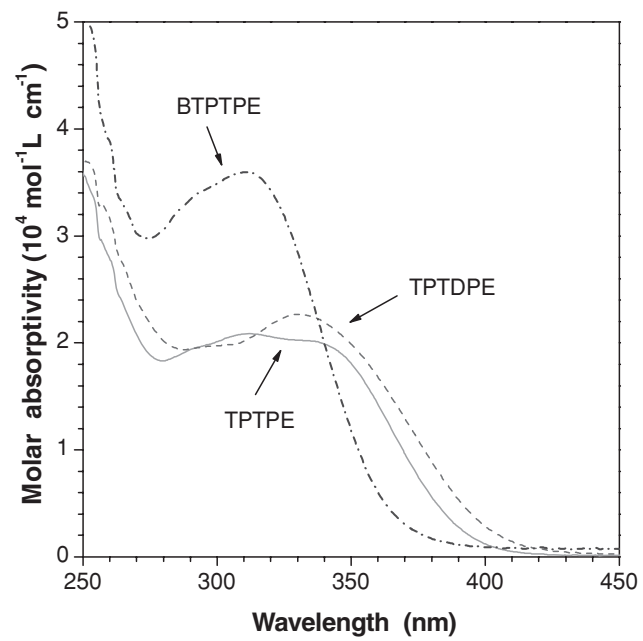

Figure 2. Absorption spectra of THF solutions of TPTPE, TPTDEP, and BTPTPE. Solution concentration: $10^{-5} \mathrm{M}$.

at a heating rate of $10{ }^{\circ} \mathrm{C} \mathrm{min}^{-1}$. All compounds exhibit clear melting transitions $\left(T_{\mathrm{m}}\right)$ in the range from 205 to $282^{\circ} \mathrm{C}$. Particularly, the second heating scan of BTPTPE detects a glass-transition temperature $\left(T_{\mathrm{g}}\right)$ at $113{ }^{\circ} \mathrm{C}$, suggesting that it possesses high morphological stability. The $T_{\mathrm{g}}$ of TPTPE and TPTDPE are not detected, probably due to their low molecular weights.

\subsection{Electroluminescence}

The efficient light emissions of TPTPE, TPTDPE, and BTPTPE encouraged us to investigate their electroluminescence (EL) properties. Multilayer OLEDs with a configuration of indium tin oxide (ITO)/ $N, N$-bis(1-naphthyl)- $N, N$-diphenylbenzidine (NPB) $(60 \mathrm{~nm}) /$ LEL $(20 \mathrm{~nm}) / 2,2^{\prime}, 2^{\prime \prime}$-(1,3,5-benzinetriyl)-tris(1phenyl-1H-benzimidazole) (TPBi) $(40 \mathrm{~nm}) / \mathrm{LiF} \quad\left(\begin{array}{ll}1 & \mathrm{~nm}\end{array}\right) / \mathrm{Al}$ a)

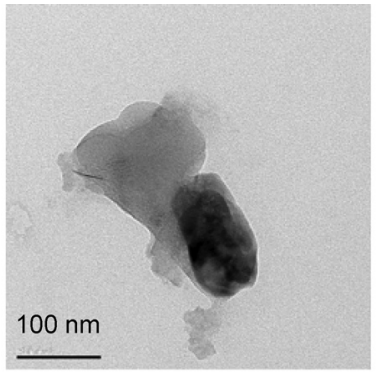

b)

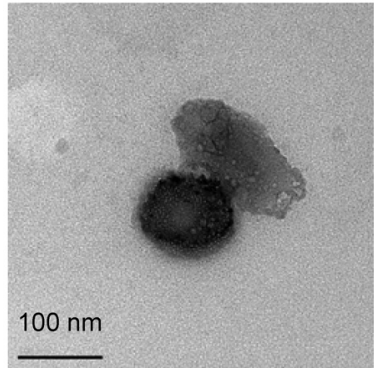

c)

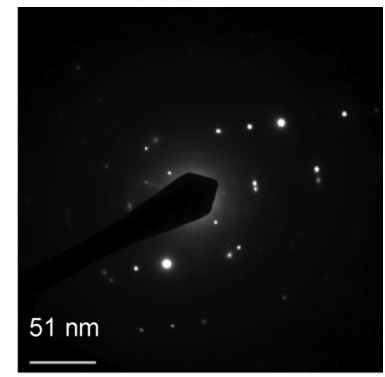

d)

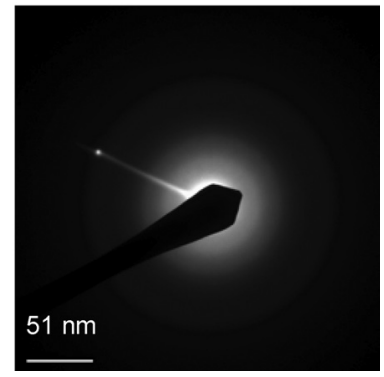

Figure 4. a,b) TEM images and (c,d) ED patterns of $(a, c)$ crystalline and $(b, d)$ amorphous aggregates of BTPTPE formed in THF/ $\mathrm{H}_{2} \mathrm{O}$ mixtures with water content of 80 and 95 vol\%, respectively.

(100 nm) were fabricated, where TPTPE, TPTDPE, or BTPTPE worked as a light-emitting layer (LEL), NPB functioned as a hole-transport material, and TPBi served as a hole-blocking as well as electron-transport layer, respectively. The EL performances of TPTPE are shown in Figure 8 as an example, while those of TPTDPE and BTPTPE are summarized in Table 3. The device of TPTPE is turned on at $4.2 \mathrm{~V}$ and emits a bright skyblue EL at $488 \mathrm{~nm}$ with luminance up to $10800 \mathrm{~cd} \mathrm{~m}^{-2}$ at $15 \mathrm{~V}$. The maximum current and external quantum efficiencies of the device reach $5.8 \mathrm{~cd} \mathrm{~A}^{-1}$ and $2.7 \%$, respectively. Although the device configuration is yet to be optimized, the good EL data
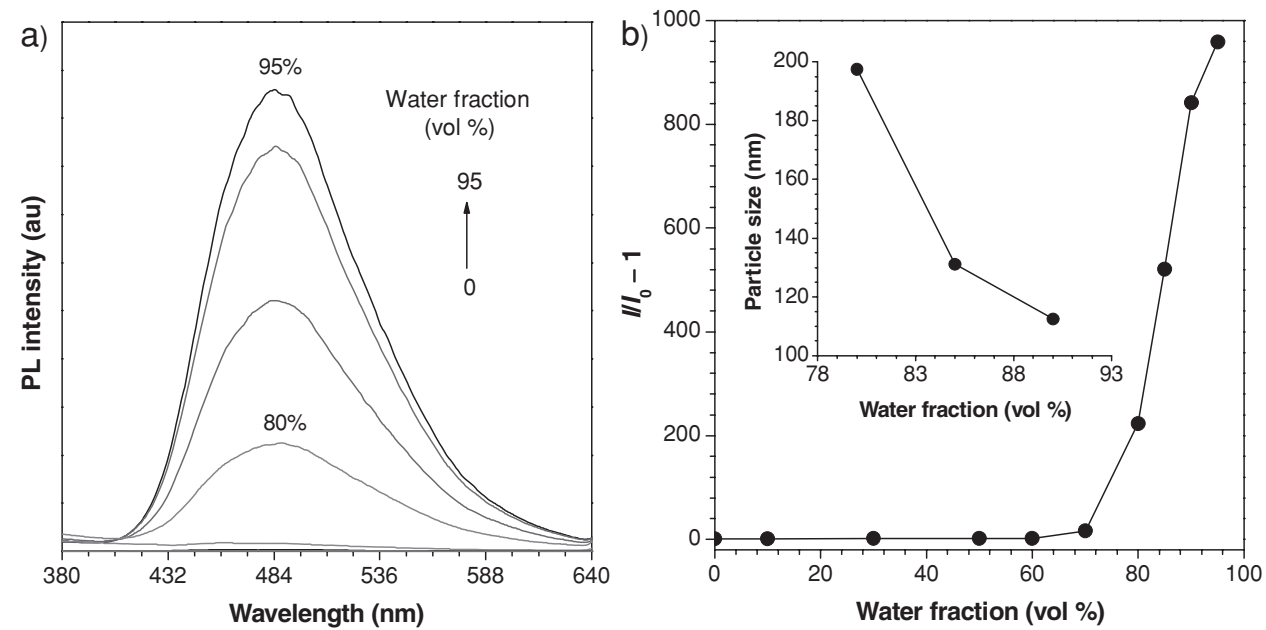

Figure 3. a) PL spectra of TPTPE in THF and THF/ $\mathrm{H}_{2} \mathrm{O}$ mixtures. Concentration: $10^{-5} \mathrm{M}$; excitation wavelength: $330 \mathrm{~nm}$. b) Plot of $\left(1 / I_{0}-1\right)$ values versus the compositions of the THF/ $\mathrm{H}_{2} \mathrm{O}$ mixtures. Inset: particle sizes of the nanoaggregates of TPTPE formed in $\mathrm{THF} / \mathrm{H}_{2} \mathrm{O}$ mixtures with various water fractions. 

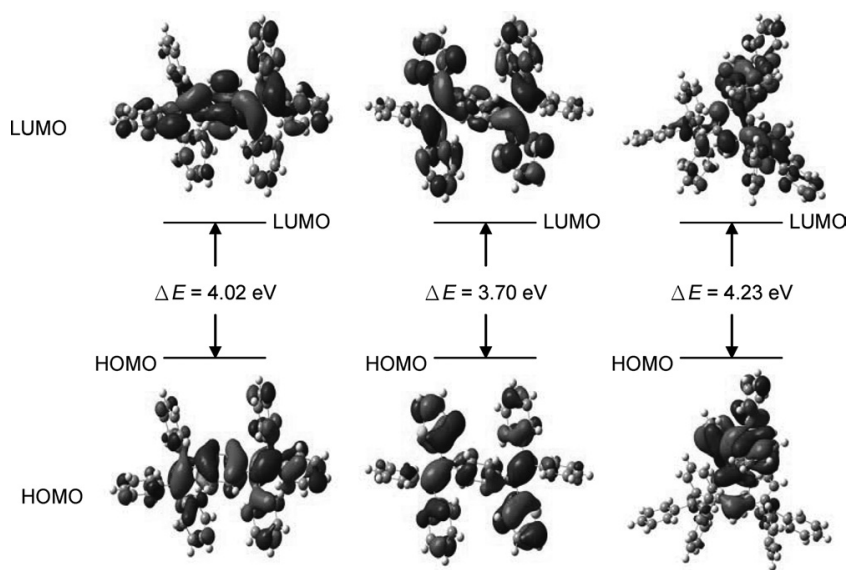

TPTPE

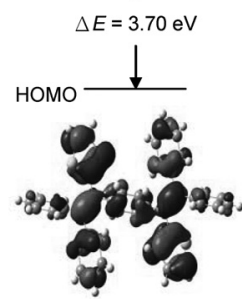

TPTDPE

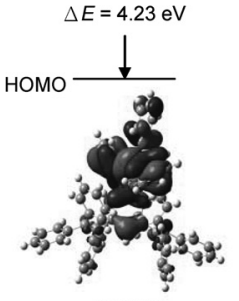

BTPTPE

Figure 5. Molecular orbital amplitude plots of $\mathrm{HOMO}$ and LUMO levels of TPTPE, TPTDPE, and BTPTPE calculated using the B3LYP/6-31G* basis set.

clearly demonstrate the high potential of TPTPE as a solid-state light emitter in the construction of efficient EL devices.

The ELs of TPTDPE and BTPTPE are observed at 512 and $448 \mathrm{~nm}$. Their EL performances, however, are much poorer than those of TPTPE. This demonstrates that the EL properties of the luminogens are sensitive to their molecular structures, which enables us to further enhance their device performances by molecular engineering.

\subsection{Explosive Detection}

Efficient fluorescent chemosensors for nitroaromatic explosives such as 2,4-dinitrotoluene, 2,4,6-trinitrotoluene, and 2,4,6trinitrophenol (picric acid; PA) are in great demand because of the threat from the increased use of explosives in terrorism acts. ${ }^{[17]}$ Inspired by the AIE characteristics of TPTPE, TPTDPE, and BTPTPE, we explored their utility as chemosensors for sensitive detection of explosives. Their nanoaggregates in $\mathrm{THF} / \mathrm{H}_{2} \mathrm{O}$ mixtures (1:9 by volume) are utilized as probes for the detection. PA was chosen as a model compound because of its commercial availability. We investigated the quenching process by monitoring the PL change in response to the PA addition.

Table 2. Optical properties of TPTPE, TPTDPE, and BTPTPE in solution $(\text { Soln })^{\text {a) }}$, aggregate (Aggr) ${ }^{\text {b) }}$, crystalline (Cryst), and amorphous (Amor) states.

\begin{tabular}{lccccc}
\hline Compound & $\lambda_{\mathrm{ab}}{ }^{\mathrm{c}}[\mathrm{nm}]$ & \multicolumn{4}{c}{$\left.\lambda_{\mathrm{em}}{ }^{\mathrm{d})}[\mathrm{nm}]\left(\Phi_{\mathrm{F}}{ }^{\mathrm{e}}\right)[\%]\right)$} \\
\cline { 3 - 6 } & & $\left.\mathrm{Soln}^{\mathrm{f}}\right)$ & Aggr & Amor & Cryst \\
\hline TPTPE & 326 & nd $(0.22)$ & 485 & $480(100.0)$ & 455 \\
TPTDPE & 332 & nd $(0.11)$ & 495 & $492(25.5)$ & 435 \\
BTPTPE & 313 & nd $(0.09)$ & 460 & $450(100.0)$ & 438 \\
\hline
\end{tabular}

a) In dilute THF solution (10 $\mu \mathrm{M})$; ${ }^{\text {b) }} \mathrm{In} \mathrm{THF} / \mathrm{H}_{2} \mathrm{O}$ mixture (1:9 by volume); c)Absorption maximum in THF; d) Emission maximum; e) Fluorescence quantum yield determined using 9,10-diphenylanthracene $\left(\Phi_{\mathrm{F}}=90 \%\right.$ in cyclohexane) as standard (for solution sample) or measured by a calibrated integrating sphere (for solid sample); ${ }^{f)}$ nd $=$ not determined because of the weak PL signals.
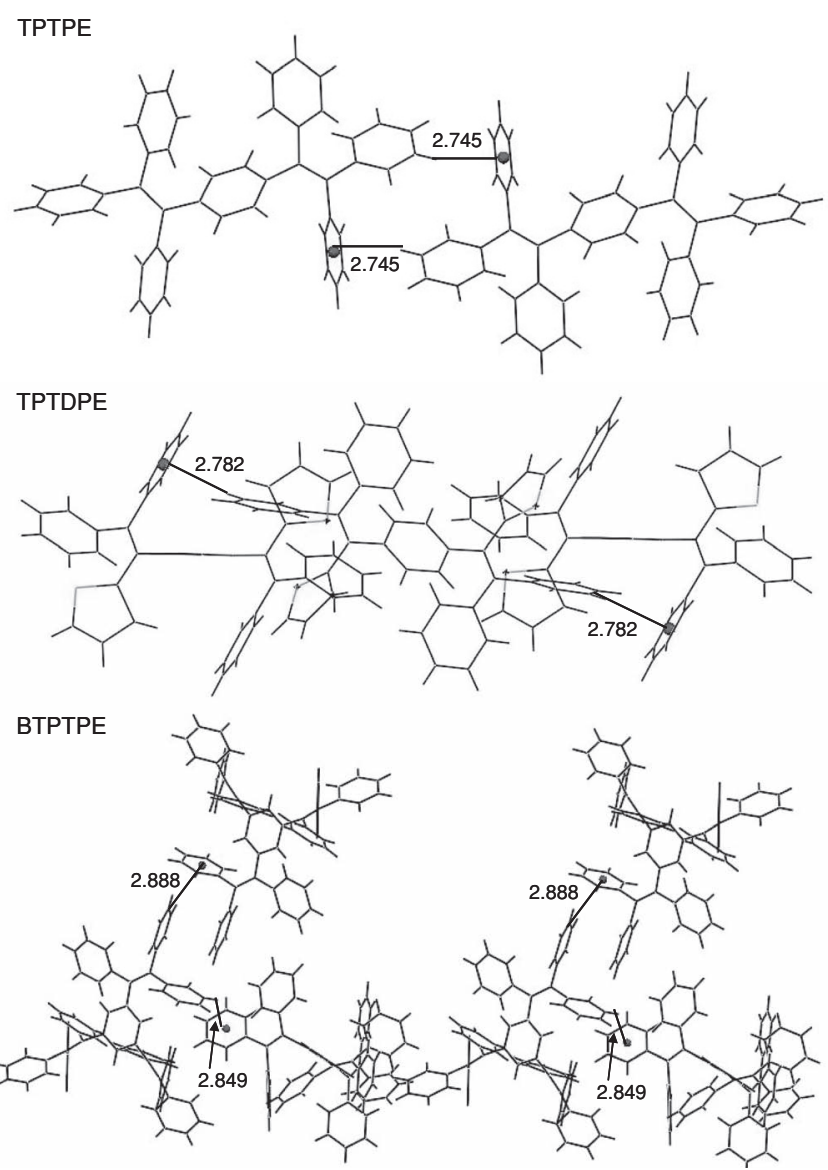

Figure 6. Perspective view of the packing arrangements in crystals of TPTPE, TPTDPE, and BTPTPE. The aromatic $\mathrm{C}-\mathrm{H} \cdots \pi$ hydrohen bonds are denoted by dotted lines.

As depicted in Figure 9, the emissions of the luminogens are progressively weakened when an increasing amount of PA is added to their $\mathrm{THF} / \mathrm{H}_{2} \mathrm{O}$ mixtures. The fluorescence quenching can be clearly discerned at a $[\mathrm{PA}]$ as low as $1 \mu \mathrm{g} \mathrm{mL}^{-1}$ or $1 \mathrm{ppm}$. At a PA concentration of $12 \mu \mathrm{g} \mathrm{mL} \mathrm{m}^{-1}$, there are almost no PL signals, which reveals their high sensitivity towards PA detection.

Remarkably, all the Stern-Volmer plots of relative PL intensity $\left(I_{0} / I\right)$ versus PA concentration give curves bending upward, instead of linear lines (Figure 9c). This indicates that PL quenching becomes more efficient with increasing quencher concentration. We have recently shown that the static quenching model was more appropriate than the diffusion-controlled dynamic mechanism to describe the PL annihilation of hyperbranched conjugated polymers by PA analytes. ${ }^{[18]}$ Such a rationalization may also apply here. TPTPE, TPTDPE, and BTPTPE can somewhat act as electron donors and have electrostatic interaction or charge transfer with PA, which leads to the formation of nonemissive ground-state dark complexes but maintaining the natural lifetimes of their unbound luminogens. However, in the diffusion-controlled dynamic quenching model, the PL lifetime is shortened with an increase in the quencher 

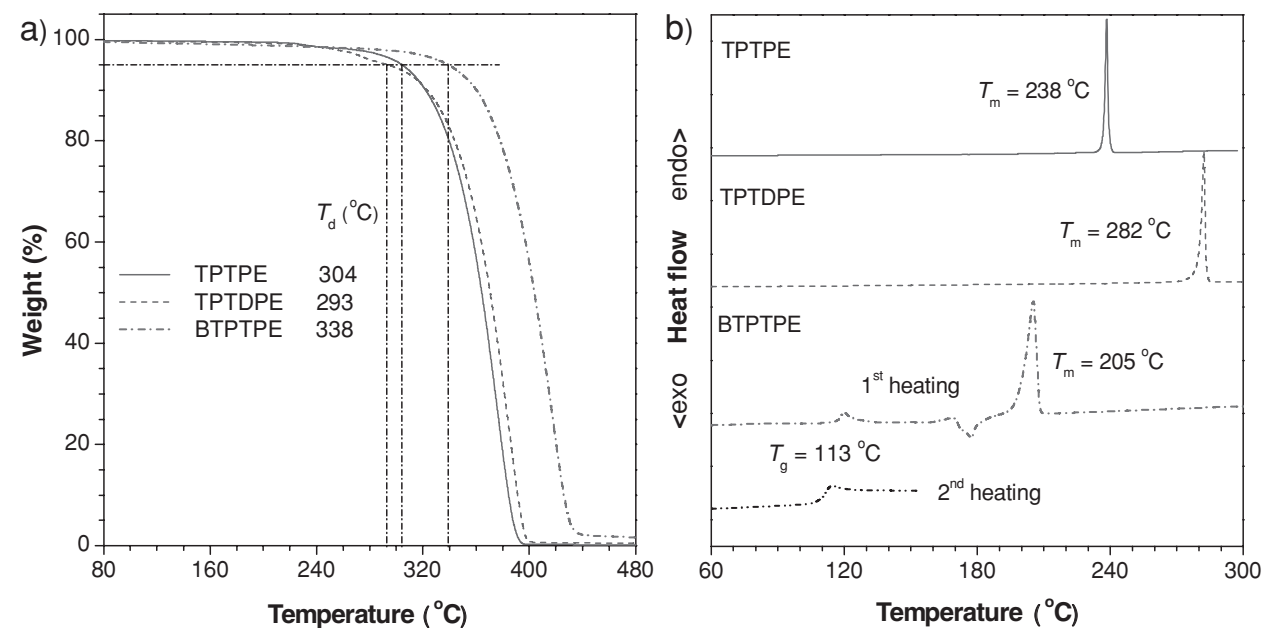

Figure 7. a) TCA and b) DSC thermograms of TPTPE, TPTDPE, and BTPTPE recorded under nitrogen at a heating rate of $10^{\circ} \mathrm{C} \mathrm{min}^{-1}$.

concentration. ${ }^{[19]}$ To verify which model is responsible for the PL quenching process of the present luminogens, the dependence of their lifetimes on the PA concentration is investigated. As shown in Figure S2 and Table S4 in the Supporting Information, the weighted mean lifetimes of the dyes remain almost unchanged in the presence of different amounts of PA, which suggests that PL quenching is through the static mechanism.

Equation 1 can be used to describe the PL annihilation by the static mechanism:

$\frac{I_{0}}{I}=e^{V_{\mathrm{q}}[\mathrm{PA}]}$

where $I_{0}$ and $I$ are the PL intensities in the absence and presence of PA, respectively, and $V_{\mathrm{q}}$ is the static quenching constant in unit of $\mathrm{L} \mathrm{mol}^{-1} .^{[19]}$ By fitting the Stern-Volmer plots shown in Figure 9c, Equations 2, 3, and 4 are obtained for TPTPE, TPTDPE, and BTPTPE in aqueous mixtures with $90 \%$ water contents, respectively.
$\frac{I_{0}}{I}=1.40 e^{46157[\mathrm{PA}]}-0.35$

$\frac{I_{0}}{I}=2.85 e^{37029[\mathrm{PA}]}-1.86$

$\frac{I_{0}}{I}=1.08 e^{61708[\mathrm{PA}]}-0.02$

Generalization from Euation 2 to 4 gives Equation 5, which is different from Equation 1 by having two extra constants (A and B), although they are both single exponential-growth functions.

$\frac{I_{0}}{I}=A e^{k[\mathrm{PA}]}+B$

If we conservatively neglect these two terms, the $k$ value in Equation 5 becomes the static quenching constants, equal to 46157,37029 , and $61708 \mathrm{~L} \mathrm{~mol}^{-1}$ for TPTPE, TPTDPE, and BTPTPE, respectively. These values are much higher than those $\left(1-185 \mathrm{~L} \mathrm{~mol}^{-1}\right)$ of linear iptycene-containing
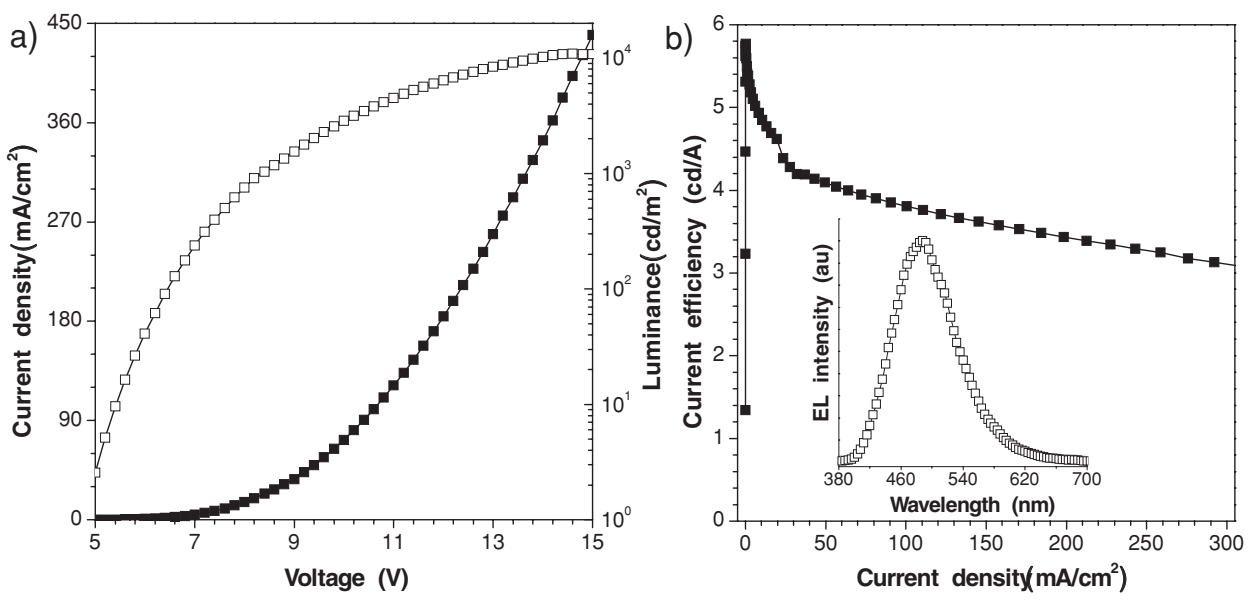

Figure 8. Changes in a) current density and luminance with the applied voltage and b) current efficiency with the current density in a multilayer lightemitting diode of TPTPE with a device configuration of ITO/NPB/TPTPE/TPBi/LiF/Al. Inset: EL spectrum of TPTPE. 
Table 3. EL performances of TPTPE, TPTDPE, and BTPTPE. With a device configuration of ITO/NPB/LEL/TPBi/LiF/Al. Abbreviations: LEL = light-emitting layer, $\lambda_{\mathrm{EL}}=$ electroluminescence maximum, $V_{\mathrm{on}}=$ turn-on voltage at $1 \mathrm{~cd} \mathrm{~m}^{-2}, L_{\max }=$ maximum luminance, $\eta_{\mathrm{P}}=$ maximum power efficiency, $\eta_{\mathrm{C}}=$ maximum current efficiency, and $\eta_{\text {ext }}=$ maximum external quantum efficiency.

\begin{tabular}{lccccccc}
\hline No. & LEL & $\begin{array}{c}\lambda_{\mathrm{EL}} \\
{[\mathrm{nm}]}\end{array}$ & $\begin{array}{c}V_{\text {on }} \\
{[\mathrm{V}]}\end{array}$ & $\begin{array}{c}L_{\max } \\
{\left[\mathrm{cd} \mathrm{m}^{-2}\right]}\end{array}$ & $\begin{array}{c}\eta_{\mathrm{P}} \\
{\left[\mathrm{m} \mathrm{W}^{-1}\right]}\end{array}$ & $\begin{array}{c}\eta_{\mathrm{C}} \\
{\left[\mathrm{cd} \mathrm{A}^{-1}\right]}\end{array}$ & $\begin{array}{c}\eta_{\text {ext }} \\
{[\%]}\end{array}$ \\
\hline 1 & TPTPE & 488 & 4.2 & 10800 & 3.5 & 5.8 & 2.7 \\
2 & TPTDPE & 512 & 4.2 & 7620 & 2.2 & 3.0 & 1.1 \\
3 & BTPTPE & 448 & 5.2 & 3530 & 1.4 & 2.8 & 1.6 \\
\hline
\end{tabular}

poly( $p$-phenylenebutadiynylene)s and poly( $p$-phenyleneethynylene)s, widely investigated fluorescent polymers for explosive detection. ${ }^{[20]}$ At low quencher concentrations $([\mathrm{PA}] \rightarrow 0)$, Equation 5 is readily converted to Equation 6 by a mathematical treatment of the Taylor expansion, which can be reorganized to give Equation 7 where $K=A k, C=A+B=1$.

$\frac{I_{0}}{I}=A\left(1+k[\mathrm{PA}]+\frac{1}{2} k^{2}[\mathrm{PA}]^{2}+\cdots+\frac{k^{n}[\mathrm{PA}]^{n}}{n !}+\cdots\right)+B$
$\frac{I_{0}}{I}=A(1+k[\mathrm{PA}])+B=A k[\mathrm{PA}]+A+B=K[\mathrm{PA}]+C$

Thus, the static quenching constants $(K)$ for TPTPE, TPTDPE, and BTPTPE at the initial stage of the SternVolmer plots are 64620, 105533, and $66645 \mathrm{~L} \mathrm{~mol}^{-1}$, respectively, which are much higher than those $\left(K<20000 \mathrm{~L} \mathrm{~mol}^{-1}\right)$ of the fluorescent chemosensors based on linear polysiloles. ${ }^{[21]}$

Generally, intrinsic autoaggregation and/or analyteinduced aggregation cause self-quenching problems that greatly reduce the sensing performance. However, aggregation is beneficial to the PL of the dye molecules. The PA molecules in the inner cores and on the outer shells of the aggregates work cooperatively to facilitate PL annihilation through electron and/or energy transfers, thus making the emission quenching a highly efficient process. By plotting the relative $\mathrm{PL}$ intensity versus $e^{k[\mathrm{PA}]}$, linear relationships are obtained (Figure 9d), which enable quantitative analysis. We are now fabricating thin-film-based chemosensors and examining their sensitivity and selectivity to various explosives.
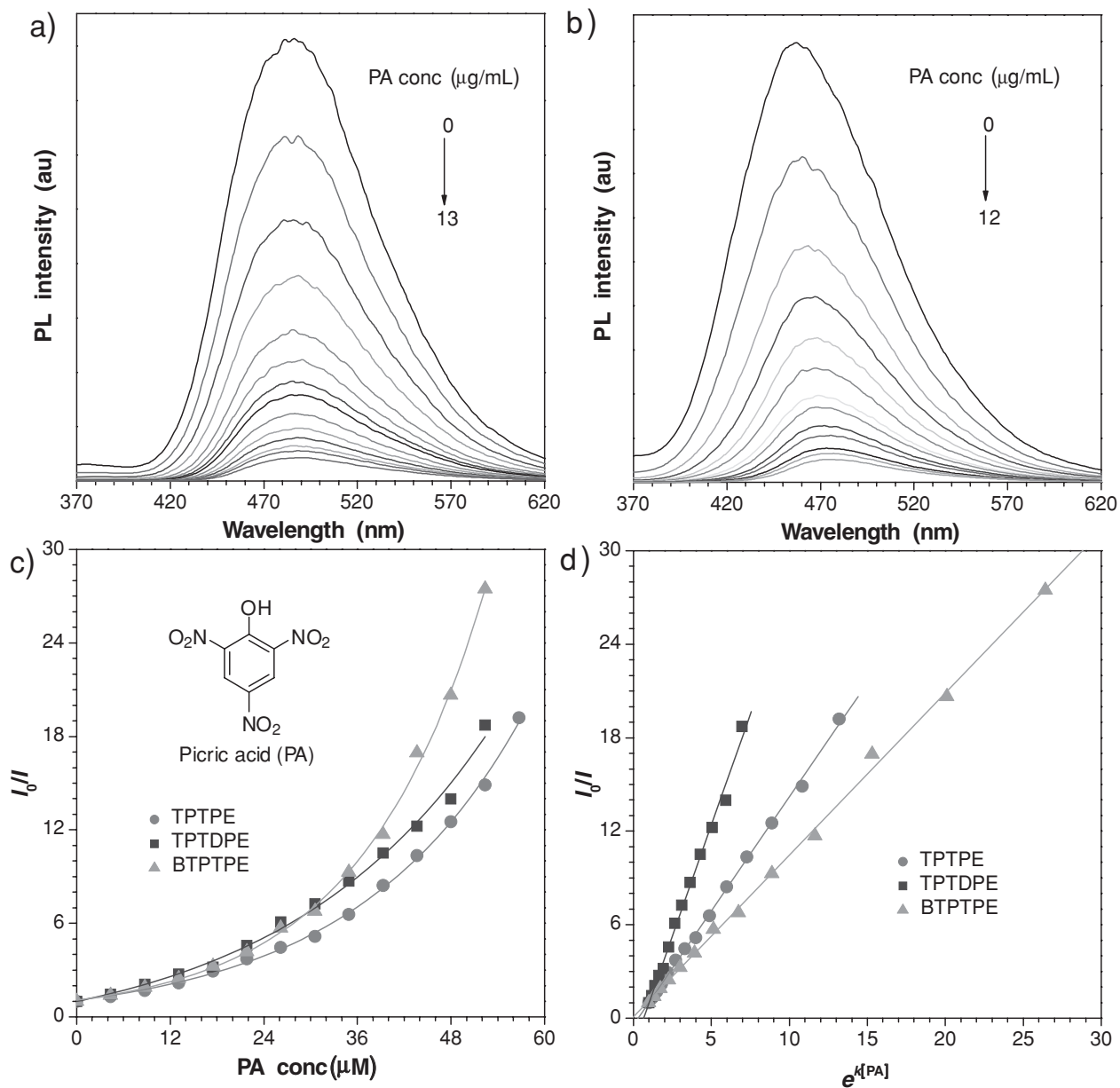

Figure 9. PL spectra of a) TPTPE and b) BTPTPE in THF/ $\mathrm{H}_{2} \mathrm{O}$ mixtures (1:9 by volume) containing different amounts of picric acid (PA). Concentration: $10^{-5} \mathrm{M}$; excitation wavelength: $330 \mathrm{~nm}$. Plots of relative PL intensities $\left(I_{0} / I\right)$ versus C) PA concentrations, and d) $e^{k[P A]}$ values of TPTPE, TPTDPE, and BTPTPE in THF $/ \mathrm{H}_{2} \mathrm{O}$ mixtures (1:9 by volume). $I_{0}=$ intensity at $[\mathrm{PA}]=0 \mu \mathrm{M}$. 

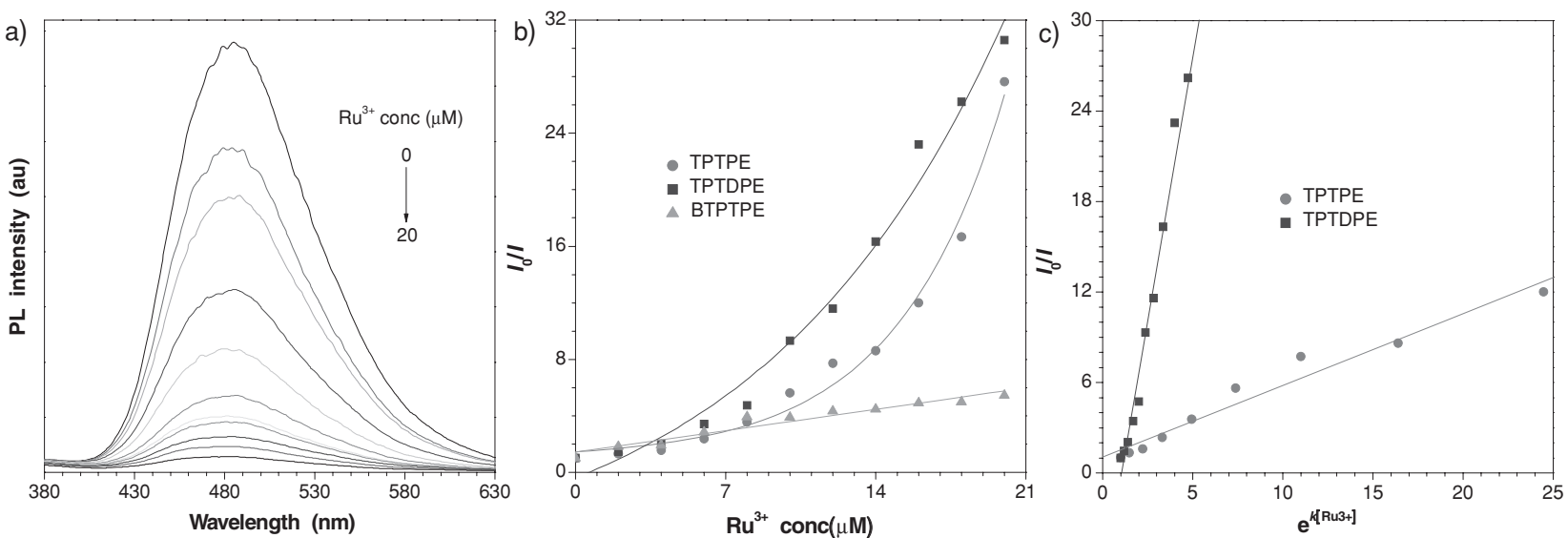

Figure 10. a) PL spectra of TPTPE in THF/ $\mathrm{H}_{2} \mathrm{O}$ mixtures (1:9 by volume) with varied $\mathrm{Ru}^{3+}$ concentrations. Plots of relative $\mathrm{PL}$ intensities $\left(I_{0} / l\right)$ versus b) $\mathrm{Ru}^{3+}$ concentrations and c) $e^{k[R u 3+]}$ values of TPTPE, TPTDPE, and BTPTPE in THF $/ \mathrm{H}_{2} \mathrm{O}$ mixtures (1:9 by volume). $I_{0}=$ intensity at $\left[R u^{3+}\right]=0 \mu \mathrm{m}$.

\subsection{Metal-Ion Sensors}

The development of highly selective sensors for metal ions is particularly important because metal ions can have detrimental effects on human and the environment. Ruthenium(III) complexes are used widely as catalysts in oxidation and metathesis reactions, but they are corrosive and destructive to the respiratory tract, eyes, skin, and digestive tract. They are regarded as a risk to aquatic organisms and may cause long-term adverse effects on the aquatic environment. Thus, the monitoring of ruthenium(III) ion, one of the most common and stable forms of ruthenium, is becoming an important issue. However, ratiometric fluorescent probes for $\mathrm{Ru}^{3+}$ are rare at present. Thus, we investigated whether the present luminogens can serve as sensitive and selective fluorescent chemosenors for $\mathrm{Ru}^{3+}$ ion detection.

With the gradual addition of $\mathrm{Ru}^{3+}$ ion to the nanoparticle suspensions of the luminogens in $\mathrm{THF} / \mathrm{H}_{2} \mathrm{O}$ mixtures (1:9 by volume), the emissions of TPTPE, TPTDPE, and BTPTPE decrease progressively (Figure 10). The PL quenching can be clearly recognized at a low $\mathrm{Ru}^{3+}$ concentration of $1 \mu \mathrm{g} \mathrm{mL} \mathrm{L}^{-1}$. At $\left[\mathrm{Ru}^{3+}\right]=20 \mu \mathrm{M}$, the PL intensity of BTPTPE is merely $\sim 30 \%$ of its original value. At the same ion concentration, in contrast, the emissions of TPTPE and TPTDPE are almost quenched completely, showing a 10-fold higher sensitivity. The SternVolmer plots of $I_{0} / I$ values versus $\mathrm{Ru}^{3+}$ concentrations for TPTPE and TPTDPE give exponential growth curves similar to those in Figure 9c, which indicates that the PL quenching process is more efficient at higher quencher concentration.

TPTPE, TPTDPE, and BTPTPE are considered to be electronrich, while $\mathrm{Ru}^{3+}$ is electron-deficient. Thus, electrostatic interaction or charge-transfer complexation may play an important role for the PL annihilation. As mentioned previously, in the static quenching model, the luminogenic molecules that bind to the quencher molecules are in the nonemissive or "dark" state, while the unbound molecules exhibit their intrinsic lifetimes. Thus, we measured the lifetimes of the luminogens in the absence and presence of quencher to check whether the same mechanism operates for the metal-ion-caused emission quenching. TPTPE in the aggregate state was chosen as an example (Figure S3 in the Supporting Information). The lifetime of TPTPE in $90 \%$ aqueous mixture remains unchanged when different amounts of $\mathrm{Ru}^{3+}$ are added, which suggests that the PL quenching process operates through the static mechanism. As a result, we obtained the following equations (Equations 8 and 9) for TPTPE and TPTDPE:

$\frac{I_{0}}{I}=0.47 e^{199868[\mathrm{PA}]}+0.99$

$\frac{I_{0}}{I}=6.98 e^{86495[\mathrm{PA}]}-7.36$

Similar to the case for explosive detection, the $k$ values for TPTPE and TPTDPE are 199868 and $86495 \mathrm{~L} \mathrm{~mol}^{-1}$, respectively, if we neglect the two constants as in Equation 5 . When the $\mathrm{Ru}^{3+}$ concentration is very low, the static quenching constants $(K)$ at the initial stage of the Stern-Volmer plots become 94390 and $603785 \mathrm{~L} \mathrm{~mol}^{-1}$, respectively.

To evaluate whether $\mathrm{Ru}^{3+}$ detection is selective, we studied the PL change of the luminogens in the presence of other metal ions. As shown in Figure 11, addition of other metal ions, including $\mathrm{Mg}^{2+}, \mathrm{Fe}^{2+}, \mathrm{Fe}^{3+}, \mathrm{Co}^{2+}, \mathrm{Ni}^{2+}, \mathrm{Cu}^{2+}, \mathrm{Zn}^{2+}, \mathrm{Rh}^{3+}, \mathrm{Ag}^{+}$, and $\mathrm{Cd}^{2+}$, exerts little change on the PL of TPTPE, TPTDPE, and BTPTPE, which indicates the high selectivity of the three luminogens towards $\mathrm{Ru}^{3+}$. Although the reason for such selectivity remains unclear at present, we believe that the higher standard reduction potential of the $\mathrm{Ru}(\mathrm{III}) / \mathrm{Ru}(0)$ couple relative to other cation/metal systems may be responsible for such selective sensing.

\section{Conclusion}

We have designed and synthesized a group of benzene-cored luminogens with multiple triarylvinyl peripheries. All the luminogens were weakly emissive in solution but became strong emitters when aggregated in the condensed phase, which is demonstrative of the novel phenomenon of AIE. The benzene-cored luminogens exhibit high solid-state fluorescence quantum yields and enjoy high thermal and morphological 

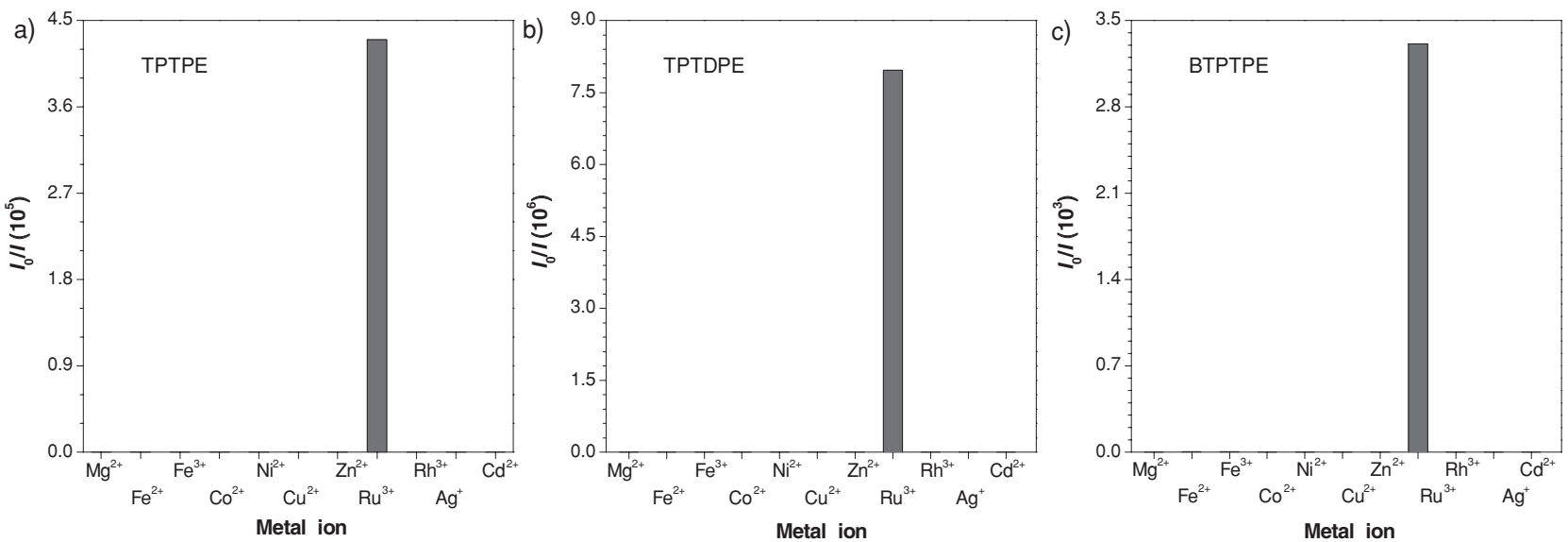

Figure 11. Changes in relative PL intensities ( $\left.I_{0} / I\right)$ of a) TPTPE, b) TPTDPE, and c) BTPTPE in THF/ $\mathrm{H}_{2} \mathrm{O}$ mixtures (1:9 by volume; $\left.10 \mu \mathrm{m}\right)$ with various metal ions $(2 \mathrm{~mm}) . I_{0}=$ intensity in the absence of metal ions.

stabilities. OLEDs using TPTPE, TPTDPE, and BTPTPE as emitters were fabricated, and show high luminance and current and external quantum efficiencies; up to $10800 \mathrm{~cd} \mathrm{~m}^{-2}, 5.8 \mathrm{~cd} \mathrm{~A}^{-1}$, and $2.7 \%$, respectively. The luminogen emissions could be quenched efficiently by $\mathrm{PA}$ and $\mathrm{Ru}^{3+}$ with large quenching constants, which renders the luminogens promising as chemosensors for explosives and metal ions.

\section{Experimental Section}

Materials and Instrumentation: DCM and THF were distilled under nitrogen from calcium hydride and sodium benzophenone ketyl, respectively, immediately prior to use. All chemicals were purchased from Aldrich and used as received. ${ }^{1} \mathrm{H}$ and ${ }^{13} \mathrm{C}$ NMR spectra were measured on a Bruker AV 300 spectrometer in deuterated chloroform using tetramethylsilane (TMS; $\delta=0$ ) as internal reference. High-resolution mass spectra (HRMS) were recorded on a GCT premier CAB048 mass spectrometer operating in MALDI-TOF mode. UV spectra were measured on a Milton Roy Spectronic 3000 Array spectrophotometer. PL was recorded on a Perkin-Elmer LS 55 spectrofluorometer. TCA was carried on a TA TGA Q5000 under nitrogen at a heating rate of $10{ }^{\circ} \mathrm{C} \mathrm{min}$. Thermal transitions of the luminogens were investigated by DSC using a TA DSC Q1000 under dry nitrogen at a heating rate of $10^{\circ} \mathrm{C} \mathrm{min}^{-1}$. TEM images and electron diffraction patterns were taken on a JEOL $100 \mathrm{CX}$ TEM instrument. Particle sizes of the aggregates in THF/water mixtures were measured on a BeCoulter Delsa 440SX Zeta potential analyzer. Single-crystal X-ray diffraction data were collected at $100 \mathrm{~K}$ on a BrukerNonices Smart Apex CCD diffractometer with graphite monochromated Mo K $\alpha$ radiation. Processing of the intensity data was carried out using the SAINT and SADABS routines, and the structure and refinement were conducted using the SHELXL suite of X-ray programs (version 6.10). The ground-state geometries were optimized using the density functional (DFT) with B3LYP hybrid functional at the basis-set level of $6-31 \mathrm{C} *$. All calculations were performed using the Gaussian 03 package. Timeresolved PL spectra were measured by using a Hamamatsu model C4334 streak camera coupled to a spectrometer. A femtosecond titaniumsapphire oscillator was used as the excitation source. A UV beam with a wavelength of $267 \mathrm{~nm}$ (third harmonic of the laser output at $800 \mathrm{~nm}$ ) was used as the pumping source in the experiments. Pulse width and repetition rate of the laser were $200 \mathrm{fs}$ and $76 \mathrm{MHz}$, respectively. Excitation power was about $0.3 \mathrm{~mW}$. The time resolution was $20 \mathrm{ps}$. The $\mathrm{PL}$ signals were measured at $380 \mathrm{~nm}$. The decay in the PL intensity (I) with time $(t)$ was fitted by a double-exponential function (Equation 10):
$I=A_{1}^{e-t / \tau_{1}}+A_{2} e^{-t / \tau_{2}}$

where $\tau_{1}$ and $\tau_{2}$ are the lifetimes of the shorter- and longer-lived species, respectively, and $A_{1}$ and $A_{2}$ are their respective amplitudes. The weighted mean lifetime $\langle t>$ was calculated according to Equation 11:

$<\tau>=\left(A_{1} \tau_{1}+A_{2} \tau_{2}\right) /\left(A_{1}+A_{2}\right)$

Device Fabrication: The devices were fabricated on $80 \mathrm{~nm}$ ITO coated glasses with a sheet resistance of $25 \Omega \square^{-1}$. Prior to loading into the pretreatment chamber, the ITO-coated glasses were soaked in ultrasonic detergent for $30 \mathrm{~min}$, followed by spraying with deionized water for $10 \mathrm{~min}$, soaking in ultrasonic deionized water for $30 \mathrm{~min}$, and oven baking for $1 \mathrm{~h}$. The cleaned samples were treated by perfluoromethane plasma with a power of $100 \mathrm{~W}$, a gas flow of $50 \mathrm{sccm}$, and a pressure of 0.2 Torr for $10 \mathrm{~s}$ in the pretreatment chamber. The samples were transferred to the organic chamber with a base pressure of $7 \times 10^{-7}$ Torr for the deposition of NPB, emitter, and TPBi, which served as hole-transport, light-emitting, hole-blocking, and electron-transport layers, respectively. The samples were then transferred to the metal chamber for cathode deposition, which was composed of LiF capped with Al. The lightemitting area was $4 \mathrm{~mm}^{2}$. The current density-voltage characteristics of the devices were measured by a HP4145B semiconductor parameter analyzer. The forward direction photons emitted from the devices were detected by a calibrated UDT PIN-25D silicon photodiode. The luminance and external quantum efficiencies of the devices were inferred from the photocurrent of the photodiode. The electroluminescence spectra were obtained by a PR650 spectrophotometer. All measurements were carried out under air at room temperature without device encapsulation.

Preparation of Nanoaggregates: Stock THF solutions of the luminogens were prepared with a concentration of $10^{-3} \mathrm{M}$. Aliquots of the stock solution were transferred to $10 \mathrm{~mL}$ volumetric flasks. After appropriate amounts of THF were added, water was added dropwise under vigorous stirring to furnish $10^{-5} \mathrm{M}$ solutions with various water contents (0-95 vol\%). PL measurements of the resulting solutions were then performed immediately.

Preparation of Metal-Ion Solutions: Inorganic salts (magnesium chloride, iron(II) chloride, iron(III) chloride, cobalt(II) chloride, nickel(II) chloride, copper(II) chloride, zinc chloride, ruthenium(III) chloride, rhodium(III) chloride, silver chloride, and cadmium chloride) were dissolved in distilled water $(10 \mathrm{~mL})$ to afford $10 \mathrm{~mm}$ aqueous solutions. The stock solutions were diluted to the desired concentrations with distilled water for further experiments.

Synthesis of 1,4-Phenylene Bis(pheny/methanone) (2): Terephthaloyl chloride (1, $8 \mathrm{~g}, 39.4 \mathrm{mmol})$, aluminium chloride $(26.3 \mathrm{~g}, 197.0 \mathrm{mmol})$, 
and benzene $(40 \mathrm{~mL})$ were added to a $100 \mathrm{~mL}$ one-necked roundbottomed flask. The reaction mixture was heated under reflux for $24 \mathrm{~h}$. A large amount of cold water was added to quench the reaction and the reaction mixture was then extracted with DCM. The organic layer was washed with water and dried over magnesium sulfate. After filtration and solvent evaporation, the crude product was purified by silica-gel column chromatography using hexane/DCM (3:2 by volume) as eluent. The product 2 was obtained as a white solid ( $6.4 \mathrm{~g} ; 56.7 \%$ yield). ${ }^{1} \mathrm{H}$ NMR $\left(400 \mathrm{MHz} \mathrm{CDCl}_{3}\right), \delta(\mathrm{ppm}): 7.89(\mathrm{~s}, 4 \mathrm{H}), 7.84(\mathrm{~d}, 4 \mathrm{H}), 7.63(\mathrm{t}, 4 \mathrm{H}), 7.51$ (t, 4H). ${ }^{13} \mathrm{C}$ NMR $\left(75 \mathrm{MHz}, \mathrm{CDCl}_{3}\right), \delta$ (ppm): 196.71, 141.32, 137.61, $133.65,130.80,130.40,129.16$. HRMS (MALDI-TOF): $\mathrm{m} / \mathrm{z} 286.1020 \mathrm{CM}^{+}$, calcd 288.0994]

Synthesis of 1,4-Phenylene Bis(2-thieny/methanone) (3): Compound 3 was synthesized from 1 (5 g, $24.6 \mathrm{mmol})$, aluminium chloride (16.4 g, $123.1 \mathrm{mmol})$, and thiophene $(5.2 \mathrm{~g}, 61.6 \mathrm{mmol})$, in freshly distilled DCM $(50 \mathrm{~mL})$ at room temperature. The procedure was similar to that described above, and afforded the product as a grey solid $(4.2 \mathrm{~g}$; yield $57.4 \%) .{ }^{7} \mathrm{H}$ NMR $\left(300 \mathrm{MHz}, \mathrm{CDCl}_{3}\right), \delta(\mathrm{ppm}): 7.97(\mathrm{~s}, 4 \mathrm{H}), 7.87(\mathrm{~d}, 2 \mathrm{H})$, $7.68(\mathrm{~d}, 2 \mathrm{H}), 7.20(\mathrm{t}, 2 \mathrm{H}) \cdot{ }^{13} \mathrm{C} \mathrm{NMR}\left(75 \mathrm{MHz}, \mathrm{CDCl}_{3}\right), \delta(\mathrm{ppm}): 188.19$, $143.89,141.80,135.97,135.70,129.77,128.91$. HRMS (MALDI-TOF): $\mathrm{m} / \mathrm{z} 299.0799\left[(\mathrm{M}+1)^{+}\right.$, calcd 299.0122].

Synthesis of 1,3,5-Benzenetris(pheny/methanone) (5): Compound 5 was prepared from 1,3,5-benzenetricarbonyl trichloride $(4,4 \mathrm{~g}, 15.1 \mathrm{mmol})$, aluminium chloride $(15.1 \mathrm{~g}, 113.0 \mathrm{mmol})$, and benzene $(40 \mathrm{~mL})$. The procedure was similar to that used for the synthesis of $\mathbf{2}$, and gave the product as a white solid (5.3 g; yield 90.6\%). ${ }^{1} \mathrm{H} \mathrm{NMR}\left(300 \mathrm{MHz}, \mathrm{CDCl}_{3}\right)$, $\delta(\mathrm{ppm}): 8.40(\mathrm{~s}, 3 \mathrm{H}), 7.85(\mathrm{~d}, 6 \mathrm{H}), 7.64(\mathrm{t}, 3 \mathrm{H}), 7.50(\mathrm{t}, 6 \mathrm{H}) .{ }^{13} \mathrm{C}$ NMR $\left(75 \mathrm{MHz}, \mathrm{CDCl}_{3}\right), \delta$ (ppm): 195.62, 138.90, 137.13, 134.80, 133.98, 130.80, 129.36. HRMS (MALDI-TOF): $m / z 391.1150\left[(\mathrm{M}+1)^{+}\right.$, calcd 391.1256].

Synthesis of 1-[4-(1,2,2-Triphenylvinyl)phenyl]-1,2,2-triphenylethene (TPTPE): $n$-Butyllithium solution (2.5 M in hexane; $8.0 \mathrm{mmol}$ ) was added to a solution of diphenylmethane $(1.34 \mathrm{~g}, 8.0 \mathrm{mmol})$ in dry THF $(40 \mathrm{~mL})$ at $0{ }^{\circ} \mathrm{C}$ under nitrogen. The resultant orange-red solution containing 4 was stirred at $0{ }^{\circ} \mathrm{C}$ for $2 \mathrm{~h}$ before $2(3.5 \mathrm{mmol})$ was added. The reaction mixture was allowed to warm to room temperature and was then stirred for a further $6 \mathrm{~h}$. The reaction was quenched by adding an aqueous solution of ammonium chloride. The organic layer was extracted three times with DCM. The organic layers were combined, washed with water, and dried over anhydrous magnesium sulfate. After solvent evaporation, the crude alcohol (containing excess diphenylmethane) was dissolved in about $50 \mathrm{~mL}$ of toluene in a $100 \mathrm{~mL}$ two-necked round bottomed flask equipped with a condenser. A catalytic amount of $p$-toluenesulfonic acid was then added and the mixture was then heated to reflux. After cooling to room temperature, the organic layer was washed twice with aqueous $\mathrm{NaHCO}_{3}$ solution $(25 \mathrm{~mL} ; 10 \%)$ and dried over anhydrous magnesium sulfate. After solvent evaporation, the crude product was purified by silica-gel column chromatography using hexane/DCM $(4: 1 \mathrm{v} / \mathrm{v})$ as eluent to afford the product as a white solid $(0.8$ g; yield $38.2 \%)$. ${ }^{1} \mathrm{H}$ NMR $\left(300 \mathrm{MHz} \mathrm{CDCl}_{3}\right), \delta(\mathrm{ppm}): 7.15-7.09(\mathrm{~m}, 20 \mathrm{H}), 7.03-7.02(\mathrm{~m}$, $10 \mathrm{H}), 6.79(\mathrm{~s}, 4 \mathrm{H}) .{ }^{13} \mathrm{C}$ NMR $\left(75 \mathrm{MHz}, \mathrm{CDCl}_{3}\right), \delta$ (ppm): 144.43, 144.40 $144.19,142.55,141.48,132.05,132.00,131.32,128.28,128.21,127.11$, 127.03. HRMS (MALDI-TOF): $m / z 586.3777$ [M+, calcd 586.2661].

TPTDPE and BTPTPE were prepared by similar synthetic procedures and their characterization data are given below:

1-\{4-[1-(2-Thienyl)-2, 2-diphenylvinyl]phenyl\}-1-(2-thienyl)-2, 2diphenylethene (TPTDPE): White Solid; yield $0.4 \mathrm{~g}, 21.2 \%$. ${ }^{1} \mathrm{H}$ NMR $\left(300 \mathrm{MHz}, \mathrm{CDCl}_{3}\right), \delta(\mathrm{ppm}): 7.24-7.16(\mathrm{~m}, 9 \mathrm{H}), 7.12-7.07(\mathrm{~m}, 9 \mathrm{H})$, $6.97(\mathrm{~d}, 8 \mathrm{H}), 6.76(\mathrm{t}, 2 \mathrm{H}), 6.51(\mathrm{~d}, 2 \mathrm{H}) .{ }^{13} \mathrm{C}$ NMR $\left(75 \mathrm{MHz}, \mathrm{CDCl}_{3}\right), \delta$ (ppm): 146.30, 143.64, 143.20, 141.94, 141.04, 133.77, 131.12, 130.97, $130.82,129.63,128.32,127.62,127.19,126.53,126.24,126.07$. HRMS (MALDI-TOF): $m / z 598.3684$ [M+, calcd 598.1789].

1-[3,5-Bis(1,2,2-triphenylvinyl)phenyl]-1,2,2-triphenylethene (BTPTPE): White solid; yield $0.7 \mathrm{~g}, 24.4 \%$. ${ }^{1} \mathrm{H}$ NMR $\left(300 \mathrm{MHz}, \mathrm{CDCl}_{3}\right), \delta(\mathrm{ppm})$ : $7.18(\mathrm{t}, 9 \mathrm{H}), 7.03(\mathrm{~d}, 18 \mathrm{H}), 6.90(\mathrm{t}, 6 \mathrm{H}), 6.84(\mathrm{t}, 6 \mathrm{H}), 6.62(\mathrm{t}, 6 \mathrm{H}), 6.49$ (s, $3 \mathrm{H}$ ). ${ }^{13} \mathrm{C} \mathrm{NMR}\left(75 \mathrm{MHz}, \mathrm{CDCl}_{3}\right), \delta$ (ppm): 144.36, 144.24, 143.85, $143.49,141.32,140.89,132.75,132.08,131.83,131.62,128.27,128.13$, $127.95,127.03,126.80$. HRMS (MALDI-TOF): $\mathrm{m} / \mathrm{z} 840.1480\left[\mathrm{M}^{+}\right.$, calcd 840.3756]

\section{Supporting Information}

Supporting Information is available from the Wiley Online Library or from the author.

\section{Acknowledgements}

This work was partially supported by the Research Project Competition of HKUST (RPC11SC09 and RPC10SC13), the Research Grants Council of Hong Kong $(604711,603509$, and HKUST2/CRF/10), the National Natural Science Foundation of China (20634020 and 20974028), and the University Grants Committee of Hong Kong (AoE/P-03/08). B.Z.T. acknowledges support from the Cao Guangbiao Foundation of Zhejiang University.

Received: August 23, 2011

Published online: November 14, 2011

[1] a) A. P. DeSilva, H. Q. N. Gunaratne, T. Gunnlaugsson, A. J. M. Huxley, C. P. McCoy, J. T. Rademacher, T. E. Rice, Chem. Rev. 1997, 97, 1515; b) W. P. Ambrose, P. M. Goodwin, J. H. Jett, A. Van Orden, J. H. Werner, R. A. Keller, Chem. Rev. 1999, 99, 2929; c) W. F. Patton, Biotechniques 2000, 28, 944.

[2] a) C.-T. Chen, Chem. Mater. 2004, 16, 4389; b) U. Lemmer, S. Heun, R. F. Mahrt, U. Scherf, M. Hopmeier, U. Siegner, E. O. Gobel, K. Müllen, H. Bassler, Chem. Phys. Lett. 1995, 240, 373.

[3] a) I. L. Medintz, H. T. Uyeda, E. R. Goldman, H. Mattoussi, Nat. Mater. 2005, 4, 435; b) M. De, P. S. Ghosh, V. M. Rotello, Adv. Mater. 2008, 20, 4225.

[4] a) J. Luo, Z. Xie, J. W. Y. Lam, L. Cheng, H. Chen, C. Qiu, H. S. Kwok, X. Zhan, Y. Liu, D. Zhu, B. Z. Tang, Chem. Commun. 2001, 1740; b) B. Z. Tang, X. Zhan, G. Yu, P. P. S. Lee, Y. Liu, D. Zhu, J. Mater. Chem. 2001, 11, 2974.

[5] a) Y. Hong, J. W. Y. Lam, B. Z. Tang, Chem. Soc. Rev. 2010, 39, in press; b) Z. Zhao, J. W. Y. Lam, B. Z. Tang, Curr. Org. Chem. 2010, 14, 2109; c) J. Liu, J. W. Y. Lam, B. Z. Tang, J. Inorg. Organomet. Polym. Mater. 2009, 19, 249; d) Y. Hong, J. W. Y. Lam, B. Z. Tang, Chem. Commun. 2009, 4332.

[6] C. J. Bhongale, C. W. Chang, C. S. Lee, E. W. G. Diau, C. S. Hsu, J. Phys. Chem. B. 2005, 109, 13472

[7] Y. T. Wu, M. Y. Kuo, Y. T. Chang, C. C. Shin, T. C. Wu, C. C. Tai, T. H. Cheng, W. S. Liu, Angew. Chem. Int. Ed. 2008, 47, 9891.

[8] C. X. Yuan, X. T. Tao,L. Wang, J. X. Yang, M. H. Jiang, J. Phys. Chem. C. 2009, 113, 6809 .

[9] G. Qian, B. Dai, M. Luo, D. Yu, J. Zhan, Z. Zhang, M. Dongge, Z. Y. Wang, Chem. Mater. 2008, 20, 6208

[10] K. Kokado, Y. Chujo, Macromolecules 2009, 42, 1418.

[11] a) Q. Zhao, L. Li, F. Li, M. Yu, Z. Liu, T. Yi, C. Huang, Chem. Commun. 2008, 685; b) Y. You, H. S. Huh, K. S. Kim, S. W. Lee, D. Kim, S. Y. Park, Chem. Commun. 2008, 3998.

[12] a) H. Chen, J. W. Y. Lam, J. Luo, Y. Ho, B. Z. Tang, D. Zhu, M. Wong, H. S. Kwok, Appl. Phys. Lett. 2002, 81, 574; b) Y. Cao, I. D. Parker, G. Yu, C. Zhang, A. J. Heeger, Nature 1999, 397, 414; c) J. S. Kim, P. K. Ho, N. C. Greenham, R. H. Friend, J. Appl. Phys. 2000, 88, 1073; d) S. Chen, Z. Zhao, B. Z. Tang, H. S. Kwok, J. Phys. D: Appl. Phys. 2010, 43, 095101; e) Z. Zhao, C. Deng, S. Chen, J. W. Y. Lam, W. Qin, P. Lu, Z. Wang, H. S. Kwok, Y. Ma, H. Qiu, B. Z. Tang, Chem. Commun. 2011, 47, 8847; f) Y. Liu, S. Chen, J. W. Y. Lam, P. Lu, R. T. K. Kwok, F. Mahtab, H. S. Kwok, B. Z. Tang, Chem. Mater. 2011, 23, 2536; g) Z. Zhao, S. Chen, J. W. Y. Lam, Z. Wang, P. Lu, F. Mahtab, H. H. Y. Sung, I. D. Williams, Y. Ma, H. S. Kwok, B. Z. Tang, J. Mater. Chem. 2011, 21, 7210; h) W. Z. Yuan, P. Lu, 
S. Chen, J. W. Y. Lam, Z. Wang, Y. Liu, H. S. Kowk, Y. Ma, B. Z. Tang, Adv. Mater. 2010, 22, 2159.

[13] a) Y. Yu, C. Feng, Y. Hong, J. Liu, S. Chen, K. M. Ng, K. Q. Luo, B. Z. Tang, Adv. Mater. 2011, 23, 3298; b) M. Wang, G. Zhang, D. Zhang, D. Zhu, B. Z. Tang, J. Mater. Chem. 2010, 20, 1858; C) T. Sanji, K. Shiraishi, M. Tanaka, ACS Appl. Mater. Interf. 2009, 1, 270; d) Y. Liu, C. Deng, L. Tang, A. Qin, R. Hu, J. Z. Sun, B. Z. Tang, J. Am. Chem. Soc. 2011, 133, 660; e) Y. Liu, Y. Tang, N. N. Barashkov, I. S. Irgibaeva, J. W. Y. Lam, R. Hu, D. Birimzhanova, Y. Yu, B. Z. Tang, J. Am. Chem. Soc. 2010, 132, 13951;f) Y. Hong, C. Feng, Y. Yu, J. Liu, J. W. Y. Lam, K. Q. Luo, B. Z. Tang, Anal. Chem. 2010, 82, 7035 g) Y. Hong, H. Xiong, J. W. Y. Lam, M. Häußler, J. Liu, Y. Yu, Y. Zhong, H. H. Y. Sung, I. D. Williams, K. S. Wong, B. Z. Tang, Chem. Eur. J. 2010, 16, 1232.

[14] W. Wang, T. Lin, M. Wang, T.-X. Liu, L. Ren, D. Chen, S. Huang, J. Phys. Chem. B. 2011, 114, 5983.

[15] a) Z. Li, Y. Dong, B. Mi, Y. Tang, M. Häußler, H. Tong, Y. Dong, J. W. Y. Lam, Y. Ren, H. H. Y. Sun, K. S. Wong, P. Gao, I. D. Williams, H. S. Kwok, B. Z. Tang, J. Phys. Chem. B 2005, 109, 10061; b) S. Dong, Z. Li, J. Qin, J. Phys. Chem. B 2009, 113, 434; c) G. Yu, S. Yin, Y. Liu, J. Chen, X. Xu, X. Sun, D. Ma, X. Zhan, Q. Peng, Z. Shuai, B. Z. Tang, D. Zhu, W. Fang, Y. Luo, J. Am. Chem. Soc. 2005, 127, 6335 .

[16] a) X. Luo, J. Li, C. Li, Y. Q. Dong, Z. Liu, Z. Bo, B. Z. Tang, Adv. Mater. 2011, 23, 3261; b) Y. Dong, J. W. Y. Lam, A. Qin, J. Sun, J. Liu, Z. Li, J. Sun, H. H. Y. Sung, I. D. Williams, H. S. Kwok, B. Z. Tang,
Chem. Commun. 2007, 40; c) H. Tong, Y. Q. Dong, M. Häußler, J. W. Y. Lam, H. H.-Y. Sung, I. D. Williams, J. Sun, B. Z. Tang, Chem. Commun. 2006, 1133.

[17] a) Y. Liu, R. C. Mills, J. M. Boncella, K. S. Schanze, Langmuir 2001, 17, 7452; b) T. H. Kim, H. J. Kim, C. G. Kwak, W. H. Park, T. S. Lee, J. Polym. Sci. Part A: Polym. Chem. 2006, 44, 2059; c) J. C. Sanchez, W. C Trogler, J. Mater. Chem. 2008, 18, 3143; d) T. Naddo, Y. Che, W. Zhang, K. Balakrishnan, X. Yang, M. Yen, J. Zhao, J. S. Moore, L. Zhang, J. Am. Chem. Soc. 2007, 129, 6978; e) M. E. Germain, M. J. Knapp, J. Am. Chem. Soc. 2008, 130, 5422; f) A. D. Hughes, I. C. Glenn, A. D. Patrick, A. Ellington, E. V. Anslyn, Chem. Eur. J. 2008, 14, 1822; g) Y. Jiang, H. Zhao, N. Zhu, Y. Lin, P. Yu, L. Mao, Angew. Chem. Int. Ed. 2008, 47, 8601; h) Y. Long, H. Chen, Y. Yang, H. Wang, Y. Yang, N. Li, K. Li, J. Pei, F. Liu, Macromolecules 2009, $42,6501$.

[18] a) J. Liu, Y. Zhong, P. Lu, Y. Hong, J. W. Y. Lam, M. Faisal, Y. Yu, K. S. Wong, B. Z. Tang, Polym. Chem. 2010, 1, 426; b) P. Lu, J. W. Y. Lam, J. Liu, C. K. W. Jim, W. Yuan, N. Xie, Y. Zhong, Q. Hu, K. S. Wong, K. K. L. Cheuk, B. Z. Tang, Macromol. Rapid Commun. 2010, 31, 834

[19] B. Valeur, Molecular Fluorescence: Principle and Applications; WileyVCH: Weinheim, Germany 2002.

[20] D. Zhao, T. M. Swager, Macromolecules 2005, 38, 9377.

[21] a) J. C. Sanchez, A. G. DiPasquale, A. L. Rheingold, W. C. Trogler, Chem. Mater. 2007, 19, 6459; b) H. Sohn, M. J. Sailor, D. Magde, W. C. Trogler, J. Am. Chem. Soc. 2003, 125, 3821. 